\title{
El aprendizaje ubicuo y el futuro de la enseñanza
}

\section{Ubiquitous Learning and the Future of Teaching}

\section{L'apprentissage omniprésent et l'avenir de l'enseignement}

\author{
Nicholas C. Burbules
}

University of Illinois, Urbana-Champaign, Estados Unidos

\section{RESUMEN}

Este ensayo examina la implicaciones del aprendizaje ubicuo, en cualquier lugar o momento, para replantear la enseñanza como actividad. El estudio aborda los siguientes temas: (1) los cambios que promueven el aprendizaje como un proceso más continuo, integrado al flujo de las actividades humanas; (2) los cambios que promueven el aprendizaje como un proceso más situado y contextual; (3) los cambios que promueven un aprendizaje más reflexivo; (4) los cambios que promueven un aprendizaje más colaborativo; (5) los cambios que promueven la enseñanza bajo un modelo más orientado a la asociación con los estudiantes; (6) cambios que integran aprendizajes de tipo formal, informal, situado y experiencial; (7) cambios que promueven nuevas relaciones con otros actores del proceso de aprendizaje (padres, lugares de trabajo, y más); y finalmente (8) cambios que promueven oportunidades para el aprendizaje ubicuo de los mismos profesores. Cada uno de estos cambios representa un conjunto de oportunidades y desafíos para enseñar y aprender de nuevas maneras. El ensayo concluye con algunas breves observaciones acerca de las repercusiones de estos cambios para la preparación y el desarrollo profesional de los profesores.

Descriptores: Aprendizaje ubicuo; proceso de aprendizaje; aprendizaje como proceso contínuo.

\section{ABSTRACT}

This essay examines the implications of anywhere/anytime, or "ubiquitous" learning for rethinking teaching as an activity. The essay touches on the following themes: (1) changes that promote learning as a more continuous process integrated into the flow of human activities; (2) changes that promote learning as a more situated and contextual process; (3) changes that promote more reflective learning; (4) changes that promote more collaborative learning; (5) changes that promote teaching in more of a partnership model with learners; (6) changes that integrate formal learning, informal learning, and situated, experiential learning; (7) changes 
that promote new relations with other partners in the learning process - parents, workplaces, and so on; and (8) changes that promote ubiquitous learning opportunities for teachers themselves. Each of these changes constitutes a set of opportunities, and challenges, for teaching and learning in new ways. The essay concludes with some brief observations about the implications of these changes for the professional training and development of teachers.

Key words: Ubiquitous learning; learning process; learning as a continuous process.

\section{RÉSUMÉ}

Cet essai examine les implications de l'apprentissage «omniprésent» n’importe où/n’importe quand pour repenser l'enseignement comme activité. L'essai aborde les thèmes suivants : (1) les changements qui favorisent l'apprentissage comme un processus plus continu intégré dans le courant des activités humaines; (2) les changements qui favorisent l'apprentissage comme processus plus situé et plus contextuel; (3) Les changements qui favorisent un apprentissage plus réfléchi; (4) les changements qui favorisent un apprentissage plus collaboratif; (5) les changements qui favorisent l'enseignement dans un modèle plus partenarial avec les apprenants; (6) les changements qui intègrent les apprentissages formels, informels, situés et expérientiels; (7) les changements qui favorisent de nouvelles relations avec d'autres partenaires dans le processus d'apprentissage - parents, lieu de travail, et le reste; et (8) les changements qui favorisent les occasions d'apprentissage omniprésent pour les enseignants eux-mêmes. Chacun de ces changements constitue une série d'opportunités et de défis pour enseigner et apprendre de façons nouvelles. L'essai se termine par de brèves observations au sujet des implications de ces changements pour la formation et le développement professionnel des enseignants.

Mots clés : L'apprentissage ubiquiste; le processus d'apprentissage; I'apprentissage comme processus continu.

$\mathrm{E}$ L FUTURO DE LA FORMACIÓN DOCENTE TENDRÁ QUE ABORDAR el aprendizaje ubicuo: la posibilidad de acceder a la información en cualquier lugar o cualquier momento, la interacción con pares y expertos eruditos y oportunidades estructuradas de aprendizaje desde una variedad de fuentes. La brecha entre el aprendizaje formal e informal desaparecerá. A menudo este aprendizaje será "ajustado al tiempo", anclado a las necesidades de una cuestión, un problema o una situación inmediata. Con él, el control de cuándo, dónde, cómo y por qué uno está aprendiendo estará en mayor medida en manos de los alumnos, y el enfoque motivacional de aprendizaje se reorientará desde el "aprendo ahora, (quizá) lo usaré más adelante", hacia a las necesidades y propósitos que el estudiante tenga en el momento.

Por supuesto, ya hemos entrado en esta época: cualquiera que haya sacado un teléfono inteligente $\mathrm{u}$ ordenador portátil para buscar en la web la respuesta a una pregunta, o para llamar a alguien para obtener información o asesoría, o para ver un vídeo tutorial en YouTube en medio de una crisis, ya se ha convertido en un aprendiz ubicuo. Debido a la propagación de dispositivos portátiles y de redes inalámbricas, que proporciona los medios para aprender en el lugar y en el momento en que sea necesario, el aprendizaje está siendo integrado en el flujo de la actividad humana cotidiana. Por cierto, el aprendizaje en una variedad de lugares y circunstancias siempre ha sido parte de la vida humana, pero el acceso a un saber estructurado a través de la tecnología hace que el lema del "aprendizaje en la vida real" sea una realidad concreta 
a un nivel sin precedentes. Además, con la llegada de "la nube" y la posibilidad de una conectividad más o menos continua, la carga y descarga de información de todo tipo, la interacción con otras personas a un clic de distancia, la tecnología portátil incorporada en gafas, etc., la integración con la tecnología se está entretejiendo en la trama misma de la experiencia ordinaria. La llamada "realidad aumentada" hace esto directamente, con ayuda de GPS y tecnologías de localización para vincular la información exactamente con su ubicación, por ejemplo, y mediante el uso de dispositivos con cámaras y pantallas (que podrían no tener aspecto de computadoras en el futuro) que le permiten ver su entorno con datos suplementarios o imágenes adecuadas para ese contenido. Imagine que su dispositivo acceda de forma automática a la página de Wikipedia sobre edificios famosos mientras usted lo está viendo a través de un visor, o que acceda al perfil de Facebook de una persona a la que le tome una fotografía o encontrar el restaurante italiano más cercano a su ubicación en una nueva ciudad, junto con comentarios de los clientes y recomendaciones sobre los mejores platos del menú. Nuestras nociones de "lugar", y de lo que hace importante a un lugar, tendrán que ser repensadas. ${ }^{1}$ El lugar ya no es sólo la ubicación física, sino que se trata del lugar como un centro o base de información complementaria, vídeo, etc.

Hay muchas salvedades que añadir aquí. La primera es que el acceso a este entorno de aprendizaje ubicuo es bastante desigual: personas con ciertos recursos económicos, personas que viven en ciertas partes del mundo, personas con mejor educación, personas que viven en ciudades y así sucesivamente, ya están viviendo de este modo. Muchos otros, claramente, no lo están. ${ }^{2}$ Esta es una preocupación grave en torno a la equidad. Pero puede ser que a medida que la conectividad inalámbrica se convierta en una utilidad gratuita, verdaderamente ubicua, disponible para todo el mundo y también a medida que el tamaño y el costo de los dispositivos a través de los cuales se accede a la Internet y a los contenidos de la nube sigue bajando, más y más gente va a tener acceso a ellos. Las personas con mejor educación y mayores habilidades tecnológicas aún obtendrán mayores beneficios de ellos, pero por lo menos se asegurará un nivel básico de acceso como un piso común. Sin embargo, esta brecha sigue siendo un gran desafío para la educación.

También es importante matizar que el acceso constante acarreará sus propios problemas (no es difícil para muchos de nosotros imaginarlo) y el estado "desconectado" o "no disponible" será aún más importante en el futuro. No querremos un flujo continuo de imágenes, sonidos o datos; no desearemos estar en contacto con otras personas (o que ellos puedan estar en contacto con nosotros) en todo momento, a veces preferiremos experimentar la vida "en bruto" sin subtítulos, comentarios o información suplementaria, por supuesto. Pero el punto es que esta será una opción en el futuro, y distintas personas hacen esta elección de diferentes maneras.

Finalmente, debo aclarar que el aprendizaje ubicuo no lo es todo en el aprendizaje y no todo se puede aprender de esta manera. Seguirá habiendo lugar para los profesores y las escuelas y el estudio. Los estudiantes más jóvenes, sobre todo, deben haber construido bases profundas de aprendizaje previamente para que la mayoría de estas oportunidades de aprendizaje pueda tener un valor o significado: sigue habiendo conocimientos y habilidades que deben ser adquiridas antes de que otros aprendizajes 
puedan ocurrir. Sin embargo, incluso este tipo de oportunidades de aprendizaje formal y estructurado están cambiando con las tecnologías ubicuas y una cultura del aprendizaje distribuido socialmente, en cualquier lugar y en cualquier momento, como se observa por ejemplo, en el caso de las tareas escolares. Las escuelas deben ser conectadas de manera consciente a una serie de otros entornos de aprendizaje: el aprendizaje de la escuela sale a estos otros lugares, y el aprendizaje de estos otros lugares regresa a la escuela. Aquí, como en todo el debate, se debe considerar que los cambios tecnológicos afectan y son afectados de manera interactiva por una serie de otros cambios sociales y culturales — una cultura de la movilidad, de colaboración, de "info-entretenimiento", videojuegos, redes sociales y de comunicación a escala mundial, literalmente. No podemos (si es que alguna vez pudimos) mantener los márgenes espaciales y temporales que se adapten a nuestra conveniencia, o nuestras suposiciones sobre dónde y cuándo ocurre el aprendizaje. El sentido más amplio de control que quisiéramos mantener sobre el aprendizaje de nuestros estudiantes es ahora algo mucho más parecido a una sociedad, en la que nuestras expectativas deben adaptarse aún más a sus estilos de aprendizaje, sus preferencias y otras opciones de acceso al aprendizaje, mucho del cual está fuera de nuestro control y podría estar fuera de nuestro alcance. Volveré sobre estas ideas en mi conclusión.

\section{II.}

¿Cómo se ve la enseñanza en un entorno de aprendizaje ubicuo? No creo que el profesor se vuelva superfluo; en cierta manera un maestro se vuelve aún más importante para los procesos de aprendizaje. Sin embargo, creo que el papel de las actividades y los objetivos de la enseñanza tendrán que cambiar y con ello, la formación del profesorado tendrá que cambiar. Esto es tanto un desafío como una oportunidad: un desafío para repensar algunas cosas, un desafío para renunciar a ciertos privilegios y autoridades tradicionales que van con nuestras ideas sobre la "enseñanza" (y que pueden ser parte de lo que nos atrajo a la docencia en la primer lugar), un reto para cambiar nuestras relaciones con los estudiantes como aprendices activos, así como con sus padres y otros. Pero al mismo tiempo, el entorno de aprendizaje ubicuo crea una serie de nuevas oportunidades para los maestros.

(1) Las universidades a menudo se refieren a la "formación continua." La era del aprendizaje ubicuo es una de "educación continua", potencialmente, para alumnos de todas las edades, desde niños hasta ancianos. Más que en secuencias de estructuras de aprendizaje, dentro de unidades espaciales y temporales que sirvan a necesidades institucionales (tanto o más de lo que sirven a las necesidades, los intereses, o los patrones de aprendizaje de los estudiantes), la sustancia del aprendizaje $-\mathrm{y}$ la predisposición a aprender - puede situarse en mayor medida en el flujo de la actividad humana. Tal como lo mencioné en un estudio anterior: Ser es aprender. ${ }^{3}$

En este punto la predisposición es un elemento crucial. La educación institucional lucha constantemente con la economía motivacional que impulsa el aprendizaje, donde las calificaciones, el rendimiento en las pruebas y la aprobación del profesor 
juegan un papel central. Por otra parte, también sabemos que estos son factores motivacionales muy pobres para muchos estudiantes.

En su lugar, podríamos pensar en maneras de transformar el lema "la sociedad del conocimiento" en una realidad concreta, donde los ciudadanos y los trabajadores, los adultos y los más jóvenes, sean criados en una cultura en la que todo tipo de actividades y experiencias, desde el trabajo al entretenimiento pasando por la vida familiar y más allá, sean vistos como oportunidades para descubrir y explorar el conocimiento adicional como complemento a dichas actividades y experiencias. El hecho de complacerse en este aprendizaje complementario puede tener su origen en las predisposiciones humanas innatas para la curiosidad y el dominio creciente de habilidades. Pero estos placeres se basan en aquellos de las propias actividades y experiencias originales, no en algo ajeno a ellas; este tipo de aprendizaje es una mejora sobre las cosas que ya son importantes para las personas.

Mi punto aquí es que la realidad emergente del aprendizaje ubicuo crea una oportunidad para que los educadores abran un diálogo largamente esperado sobre la responsabilidad compartida de crear y sostener una sociedad de aprendizaje (no sólo como un deber de las instituciones formales) y de fomentar una predisposición para aprender simplemente como parte de lo que somos como seres humanos. En lugar de luchar para motivar el trabajo y el estudio entre estudiantes que, en muchos casos, reciben mensajes contradictorios de distintas influencias sociales, una sociedad que entiende el poder y la importancia del aprendizaje ubicuo valorará diversas fuentes de aprendizaje. Esto generaría un gran recurso y un apoyo a los esfuerzos de los educadores, pero también significa, como se ha mencionado anteriormente, la pérdida de un cierto control y poder de dirección sobre el aprendizaje, en la medida que múltiples influencias y múltiples propósitos motivarán las actividades de aprendizaje.

(2) Los educadores progresistas y otros de tendencia constructivista social siempre han alabado el aprendizaje situado y contextual como uno más rico, más complejo y cuyas posibilidades de ser retenido y utilizado en el futuro son mayores. El aprendizaje ubicuo crea un recurso para apoyar el aprendizaje situado, a medida que la gente busca el conocimiento y las habilidades necesarias para hacer frente a situaciones reales e inmediatas a las que se enfrentan.

En una conocida historia del terremoto de enero 2010 en Haití, un hombre atrapado entre escombros utilizó su teléfono celular para acceder a información sobre cómo contener el sangrado de sus heridas y así ganar tiempo hasta que pudo ser rescatado, 60 horas más tarde. Aunque mucho más dramático que la situación de aprendizaje habitual, es el caso de alguien se enfrentó a un problema inmediato y tuvo un interés intrínseco - y muy poderoso - por acceder a un conocimiento que necesitaba saber. Sus lecciones tuvieron un impacto directo y él vio (y sintió) sus beneficios. ¿Habría sucedido lo mismo si hubiera tomado un taller de primeros auxilio dos años antes? Tal vez. Pero hay tanto de la formación actual del tipo "lo aprendo ahora para usarlo más adelante", que es imposible saber de antemano que elementos cambiarán la vida de una persona y cuáles nunca serán usados y pasarán al olvido. 
Hay dos puntos importantes que aclarar. En primer lugar, el caso de Haití pone de relieve un cierto valor instrumental en el aprendizaje que puede no ser aplicable a otros factores que contribuyen a mejorar la vida, pero que no se puede justificar en términos de su utilidad o impacto directo. No todo el aprendizaje ubicuo es acerca del valor de su uso, a veces puede que se trate sólo acerca del interés, la curiosidad y el enriquecimiento. El aprendizaje como complemento de la actividad y de la experiencia humana, puede tener un impacto decisivo en el camino, como puede no tenerlo - esto no quiere decir que no sea agradable o que no valga la pena. En segundo lugar, hay una cierta cantidad de aprendizaje que simplemente no se puede relegar a la necesidad y el propósito situados: un aprendizaje realmente prepara para otros aprendizajes y necesita ser desarrollado de manera explícita y sistemática a fin de que otro aprendizaje, más independiente, se produzca. Tengo la firme sospecha de que este conjunto es menor que el conjunto del que las escuelas se preocupan actualmente y, por cierto, es aún más reducido para ciertos alumnos y sus necesidades e intereses. Por lo tanto, debemos preguntarnos si la obsesión por dominar ciertos materiales en la escuela, en gran parte por razones ajenas, no podría resultar más dañina que beneficiosa para muchos estudiantes, al suprimir la predisposición a aprender que traen consigo a la escuela, pero que con el tiempo pierden.

(3) Del mismo modo, los educadores dicen que ciertas capacidades necesarias para la resolución de problemas, el pensamiento crítico, la creatividad y la investigación independiente son en sí mismos objetivos educativos importantes, además del dominio de conocimientos sobre contenidos específicos. ¿Dónde encajan estas capacidades en el plan de estudios estándar? Sabemos que en el caso de muchos estudiantes, esto nunca sucede, aún cuando dichas capacidades tienen que más con las perspectivas de vida a futuro y con el éxito que el dominio del conocimiento acerca del contenido por sí solo.

Una vez más, el aprendizaje ubicuo crea un recurso para los profesores, la oportunidad de relacionar las metas de aprendizaje con contextos y propósitos más allá del salón de clases. Aparte de los factores de predisposición y otros que ya he mencionado, una cultura de aprendizaje ubicuo considera a los eventos cotidianos como oportunidades para el aprendizaje y, ya sea que opte por tomarlas o no, la gente reconoce que cada actividad y cada experiencia están rodeadas por un halo o penumbra de información adicional. Siempre se puede decir algo más y esto cambia la forma de abordar la particularidad - ya no como un "hecho" aislado sino que siempre como un nodo de hechos interconectados y significaciones interconectadas. Reconocer esto, y aprender a navegar entre estas relaciones en red, fomenta el tipo de razonamiento del que dependen la resolución de problemas, el pensamiento crítico, la creatividad y la investigación. Precisamente porque no se puede seguir todos los caminos, el proceso de elegir cuándo y qué caminos seguir plantea cuestiones que a su vez requieren sensibilización y reflexión de segundo orden acerca de las interdependencias del conocimiento. Me permito sugerir, además, que esto sustenta también una especie de conciencia metacognitiva acerca de las propias comprensiones y presuposiciones — de dónde vienen, dónde empiezan y dónde terminan. 
(4) Además, otro aspecto del aprendizaje ubicuo, parte de la gran tendencia de la "Web 2.0", es el crecimiento de las comunidades de aprendizaje colaborativo. También en este caso los cambios tecnológicos se influyen de manera recíproca con los grandes cambios sociales y culturales. La gente considera crecientemente a la Internet como una forma de extender sus redes sociales y una manera en la que esto sucede es al co-crear y compartir texto, video, y otros productos. Los usuarios comunes parecen menos preocupados de recibir el crédito por la autoría o propiedad, y están más determinados por lo que David Weinberger llama "un espíritu de generosidad.” ${ }^{4}$ Asimismo, Yochai Benkler proclama las virtudes del "patrimonio común basado en la producción entre pares." La publicontratación ("crowdsourcing", en inglés) y otras actividades distribuidas similares surgen a partir de una identidad de comunidad no presencial y la refuerzan. La gente parece genuinamente motivada por la idea de que la inteligencia colectiva puede conseguir más que el logro de los individuos e investigadores como Benkler argumentan que tales modelos son más productivos que los sistemas de incentivos basados en el crédito personal que predominan en una variedad de lugares de trabajo. En otra ocasión he escrito acerca de la "auto-educación de las comunidades", en la que las personas toman colectivamente la responsabilidad de aprender juntos sobre las materias que les interesan. ${ }^{6}$ En estas comunidades no existen distinciones entre los roles de profesor y alumno: la gente aprende de los demás y junto con ellos; en ocasiones desempeñan el rol de expertos y profesores y en otras son aprendices bajo la tutela de los demás.

Los profesores han utilizado desde hace mucho tiempo los proyectos grupales como un modelo de trabajo en el aula y, en un sentido más amplio, a menudo para promover el valor social de la "cooperación." No obstante, los sistemas de recompensa y en general las medidas de rendición de cuentas de la escolarización en casi todos los niveles, aún premian e incentivan el logro individual como la métrica de éxito. Como se expuso anteriormente, el aprendizaje ubicuo está menos regido por tales consideraciones y se encuentra más bien en las necesidades y los objetivos de las circunstancias contextuales. En esas condiciones, la colaboración es una ventaja y, de hecho, para muchos propósitos, es indispensable. La potencialidad de un recurso ubicuo a menudo reside en las contribuciones que hacen varias personas. La información sobre un lugar que estoy visitando viene de muchos que lo visitaron antes; mi tendencia será confiar más en la sabiduría colectiva de muchas opiniones que en la de un solo individuo. Pero incluso cuando accedo a la asesoría o información de un solo perito, esto también está siendo compartido como parte de un bien colectivo y distribuido - algo que luego, a su vez, podría retribuir compartiéndolo con los demás.

Sugiero que sería provechoso que los educadores consideraran este espíritu de colaboración más amplio como un recurso educativo y una oportunidad. Una de las funciones de los profesores en este contexto es ayudar a fomentar y facilitar la creación de estas comunidades de aprendizaje distribuido (en las que podrían ser uno de muchos participantes, pero tal vez no el actor central o fundamental). Sin duda, sería beneficioso aprovechar los sistemas de motivación y recompensa que llevan a los estudiantes a querer compartir y co-crear el trabajo con los demás. Esto tal vez implicará cambiar algunas normas institucionales contra el "hacer trampa" o "no compartir el 
trabajo." Es posible que necesitemos replantear lo qué entendemos por aquellas restricciones y así cambiar, o incluso abandonar, ciertas formas tradicionales de evaluar y recompensar el trabajo del estudiante. Sin embargo, el beneficio simultáneo sería un sistema mucho más comprometido y enérgico de motivación para impulsar el aprendizaje, uno que obligaría a los educadores a pensar en estimular, fomentar y evaluar la fuerza de la colaboración como un objetivo educativo más importante en sí mismo. Como ya he dicho, muchos de nuestros alumnos ya llegaron a ese punto (y nos están esperando).

(5) Este cambio de enfoque sobre la motivación del estudiante abre otras oportunidades igualmente. En un contexto donde las habilidades y la familiaridad de los estudiantes con las nuevas tecnologías a menudo superan las del propio profesor (lo que es cada vez más evidente a medida que los estudiantes se hacen mayores), la relación de enseñanza-aprendizaje se puede plantear para que parezca más como una sociedad, en la que cada parte tiene cosas que ofrecer a la otra. El papel del profesor sigue siendo crucial, pero también sigue cambiando: mentor, facilitador, modelo a seguir, o tutor. Para llevar a cabo estas funciones de buena manera en un contexto de aprendizaje ubicuo, los maestros tienen que estar mejor informados y más comprometidos con lo que los alumnos ya saben.

Por un lado, se crea un recurso para aprovechar el nuevo aprendizaje que se relaciona con las otras experiencias contextuales y con los intereses que los alumnos traen consigo al aula; esta idea no es nueva: John Dewey ya se refería a esto hace un siglo. ${ }^{7}$ Estas relaciones son a la vez objeto del andamiaje cognitivo y de la motivación. ${ }^{8}$ Por otro lado, hacen que el cliché de "el aprendiz supera al maestro" se transforme en una realidad concreta; tanto en términos de conocimientos técnicos como de experiencias e intereses contextuales, los alumnos poseen los conocimientos y habilidades que los maestros no tienen. Esto abre el camino hacia un tema que abordaré a continuación: los profesores tienen que transformarse en estudiantes ubicuos también.

Otra forma en la que el papel del profesor sigue siendo indispensable es como un ecualizador, dado que todo el conocimiento y la experiencia que los estudiantes traen al aula, los recursos fuera de la escuela, sean tecnológicos u otros, siguen siendo profundamente desiguales. La educación pública sigue siendo el único recurso común que comparten estudiantes de todo tipo de contextos y el profesor es el punto focal para reconocer y responder las diferencias planteadas. En particular, en este ámbito, el acceso a las oportunidades de aprendizaje en línea es más que una cuestión de acceso a la tecnología en sí. No sólo se trata de una "brecha digital”; es una división de todo tipo de otras oportunidades — chances desiguales para aprender a aprovechar el acceso a la tecnología, posiblemente; desigualdad en las redes de apoyo y en las redes sociales que ayudan a fomentar las habilidades y la comprensión sobre la tecnología; desigualdad de contextos y de tiempo disponible como para experimentar y practicar con nuevos recursos tecnológicos y así sucesivamente. El aspecto más perjudicial de este tipo de división es que se está auto perpetuando e incluso acelerando; mientras más acceso a recursos ubicuos y oportunidades de aprendizaje se tiene, crecen las posibilidades de aumentar la distancia con aquellos que no lo tienen. Un mejor acceso a 
la educación es igual a un mejor acceso a oportunidades de empleo; un mejor acceso a las redes sociales, es igual a un mejor acceso a la participación ciudadana. Cada una de estas ventajas permite que la brecha entre los que tienen y los que no tienen (o entre mayor y menor nivel de acceso, cuantitativa y cualitativamente) aumente con el tiempo. En resumen, tecnológicamente hablando, los ricos se hacen más ricos. Las escuelas y sus educadores tienen una responsabilidad fundamental para tratar de contrarrestar estas tendencias, especialmente cuando se superponen sobre otras dimensiones de desigualdad educativa; los que tienen acceso ubicuo a la tecnología y a las redes en línea viven en mundos sustancialmente distintos de los que no tienen estas condiciones.

(6) Las formas radicalmente nuevas en que las personas interactúan con la tecnología en el presente también proporcionan una valiosa oportunidad para que los docentes reformulen su trabajo y su función. No es nada nuevo que gran parte de la forma en que la gente se involucra con la nueva información, a través de los medios de comunicación o a través de la navegación en línea, se base en multimedia - sobre todo en información visual y basada en video. Gran parte de la web es texto, sin duda, pero la forma en que la subida y descarga de información ocurre en contextos ubicuos a menudo sucede a través de la imagen y el sonido. De manera creciente, esperamos que nuestra tecnología - por ejemplo, el GPS — nos enseñe o nos diga lo que necesitamos saber. Esto debe ser así cuando se está transmitiendo a través de un flujo simultáneo con otras actividades, porque no se puede leer un texto extenso mientras estamos haciendo otra cosa.

Aquí, nuevamente, el caso de la realidad aumentada es instructivo: en algunos casos, esta puede ser descrita como "el mundo con subtítulos", pero cada vez en más situaciones, la realidad aumentada toma la forma de una capa adicional de experiencia, que se añade al flujo de experiencia original y está entremezclada con él. Mirando hacia el futuro, estas capas pueden ser cada vez más indistinguibles unas de otras: gran parte de la vida será una experiencia multimedia. (Imagínese caminando a través de un museo de arte, con comentarios simultáneos, acceso para ver ilustraciones de los artistas disponibles en otros museos, información visual sobre dónde y cuándo se crearon las obras de arte, por ejemplo. Piense en la posibilidad de ver una pintura superpuesta con los bocetos originales o el palimpsesto de las versiones anteriores, que fueron pintadas encima y luego alteradas)

Ayudar a los estudiantes a integrar el aprendizaje formal, el aprendizaje informal y este nuevo tipo de aprendizaje situado y experiencial ofrece, en mi opinión, una oportunidad y un recurso educativo tremendamente poderoso - uno que presenta características y desafíos verdaderamente novedosos. Un desafío aún mayor es determinar alguna forma de traer al aula los estilos de aprendizaje y las predisposiciones de una generación de aprendizaje ubicuo.

(7) Afortunadamente, los maestros no están solos en este nuevo ecosistema de atención y aprendizaje. La ubicuidad también implica nuevas asociaciones potenciales: con los padres, sin duda, pero también con otros participantes presentes en los 
distintos contextos donde el aprendizaje ubicuo sucede. La educación misma debe convertirse en un proceso en red, uno en el que la escuela y el aula sean centrales, pero no estén aisladas de otros actores. Una vez más, las consignas educativas de antaño adquieren un nuevo significado e inmediatez: la participación de los padres, el aprendizaje a lo largo y ancho de la vida, el aprender haciendo, la escuela sin muros, el aprendizaje para la vida real, el reconocimiento de que los maestros no pueden lograrlo por sí solos y así sucesivamente. Algunos de estos otros participantes pueden estar dispuestos a interactuar directamente con la sala de clases, como expertos externos, como mentores profesionales y más. En un mundo así, incluso la distinción entre aprendizaje formal e informal empieza a desaparecer y nuevos procesos, nuevas estructuras, tendrán que romper los marcos espaciales y temporales que hemos construido para distinguir dónde y cuándo deben ocurrir ciertos tipos de aprendizaje.

Esto puede parecer abrumador. No obstante, al mismo tiempo, los recursos y sistemas de apoyo para el aprendizaje se multiplican por igual. Las escuelas no son islas; el desafío educativo consiste en la vinculación y la coordinación de estos sistemas, lo que implica un tipo diferente de desafío, aunque se trata de uno que incluye elementos familiares. Todavía necesitamos teorías sobre el aprendizaje y la motivación; todavía necesitamos objetivos y normas educacionales que nos señalen lo que es importante aprender; aún necesitamos un compromiso con la equidad y el acceso para proteger nuestros valores democráticos y aún necesitamos algunas estructuras y procesos formales para regular y evaluar los tipos de aprendizaje que están teniendo lugar. El punto aquí es que es necesario replantear cada uno de estos elementos familiares de la labor del educador para un mundo de aprendizaje ubicuo y que este replanteamiento tendrá que involucrar a otros, que se convierten en socios indispensables en este proceso de articulación y coordinación de una serie de contextos de aprendizaje.

(8) Por último y quizás lo más importante, los profesores también son aprendices dentro de un entorno de aprendizaje ubicuo. Todos los recursos descritos aquí están disponibles para los profesores: el aprendizaje en cualquier lugar y en cualquier momento, el acceso móvil, las redes sociales de apoyo y asesoramiento, el aprendizaje multicontextual, la realidad aumentada, etc. Los docentes también están en medio de un flujo de oportunidades y experiencias de aprendizaje y su crecimiento y desarrollo puede ser continuo desde esta posición. Por sobre todo, los profesores pueden ser considerados como miembros de sus propias comunidades de aprendizaje distribuido, no sólo de las formas en que las redes profesionales han trabajado siempre, sino que como una característica inmediata de la vida cotidiana en sí, potencialmente disponible para el asesoramiento o el apoyo en cualquier momento de su vida laboral. Como he dicho anteriormente, los maestros no están solos.

\section{III.}

¿Cómo, entonces, enfrentan los maestros este flujo continuo de acceso a la información y a otros actores como un recurso potencial y no como una "creciente y abrumadora confusión”? ¿De qué maneras debe convertirse el aprender a manejar este flujo 
en parte de la formación profesional docente? ¿Cuáles son las nuevas habilidades que los profesores deben dominar para convertirse en facilitadores y coordinadores de múltiples fuentes y sitios de aprendizaje para sus estudiantes?

Indiquemos, primeramente, cuáles no serían: no se trata sencillamente de las habilidades de enseñar y explicar el material a los alumnos; no es sólo el dominio del conocimiento sobre los contenidos que los profesores poseen para "entregar" a los estudiantes; no son sólo las habilidades de gestión del aula y la disciplina; tampoco se trata sólo de aprender a "usar" la tecnología como un recurso pedagógico. Por supuesto que estas capacidades siguen siendo importantes, pero la formación profesional que se limita a ellas no preparará a los docentes para un mundo de aprendizaje ubicuo.

Las nuevas habilidades y capacidades de enseñanza requieren una comprensión más amplia de las redes sociales de base tecnológica y de la variedad de recursos de aprendizaje disponibles en línea, desde YouTube hasta la Khan Academy; estos requieren una comprensión sociológica y cultural de los diversos ambientes de aprendizaje y sus características; requieren nuevas teorías del aprendizaje que integren el aprendizaje formal, informal y el aprendizaje experiencial y situado; requieren habilidades en el diseño de estrategias de aprendizaje que aprovechen e interrelacionen el aprendizaje que tiene lugar en contextos diversos; además, requieren la capacidad de trabajar con un gama de socios en dichos contextos. El profesor en un mundo de aprendizaje ubicuo no es sólo un pedagogo, sino un planificador, un diseñador y un director. Esto, a su vez, sugiere una cierta ruptura en las categorías de maestro y administrador, pedagogo y planificador, profesional y diseñador y lo que los docentes necesitan saber y ser capaces de hacer.

Y, por último, estos límites borrosos plantean un reto igualmente transformador para las escuelas de educación, los organismos de acreditación y las asociaciones profesionales, acerca de revocar sus propias categorías y distinciones. En este punto, como afirmo en todo el artículo, los educadores van a tener que volver a examinar su participación en las instituciones y su interés en perpetuar una manera determinada de hacer las cosas, además de los privilegios concurrentes, los que podrían tratar de mantener, en vez adaptarse a la rápida evolución de la enseñanza y del ambiente de aprendizaje, que amenaza con hacer que esas formas establecidas de pensar y de hacer las cosas se vuelvan irrelevantes.

\section{Notas}

1. Nicholas C. Burbules, "Rethinking the virtual." The International Handbook of Virtual Learning Environments, Joel Weiss, Jason Nolan y Peter Trifonas, eds. (Dordrecht: Kluwer Publishers, 2005), pp. 3-24.

2. Nicholas C. Burbules, Thomas A. Callister, Jr., and Claudine Taaffe, "Beyond the digital divide." Technology and Education: Issues in Administration, Policy and Applications in K-12 Schools, Sharon Y. Tettegah y Richard C. Hunter, eds. (New York: Elsevier, 2006), pp. 85-99. 
3. Nicholas C. Burbules, "Meanings of ubiquitous learning." Ubiquitous Learning, Bill Cope y Mary Kalantzis, eds. (Urbana, IL: University of Illinois Press, 2009), pp. 15-20.

4. David Weinberger, "The digital future." [On-line]. Disponible: http://www.c-span. org/congress/digitalfuture.asp (2004).

5. Yochai Benkler, "Coase's penguin, or Linux and the nature of the firm." [On-line]. Disponible: http://www.benkler.org/CoasesPenguin.html (2002).

6. Nicholas C. Burbules, "Self-educating communities: Collaboration and learning through the Internet." Learning in Places: The Informal Education Reader, Zvi Bekerman, Nicholas C. Burbules, Diana Silberman-Keller, eds. (Nueva York: Peter Lang Publishing, 2006), pp. 273-284.

7. John Dewey, The School and Society, University of Chicago Press, 1915.

8. Nicholas C. Burbules y Marcia C. Linn, "Response to contradiction: Scientific reasoning during adolescence." Journal of Educational Psychology, Vol. 80 No. 1 (1988): $67-75$.

9. Burbules, Callister, Jr. y Taaffe, "Beyond the digital divide" op. cit. 